\title{
Transmission of tuberculosis in the metropolitan area of Zurich: a 3 year survey based on DNA fingerprinting
}

\author{
G.E. Pfyffer*, A. Strässle*, N. Rose+, R. Wirth*, O. Brändli+, H. Shang+
}

Transmission of tuberculosis in the metropolitan area of Zurich: a 3 year survey based on DNA fingerprinting. G.E. Pfyffer, A. Strässle, N. Rose, R. Wirth, O. Brändli, H. Shang. CERS Journals Ltd 1998.

ABSTRACT: Between 1991 and 1993, 444 inhabitants of the metropolitan area of Zurich were reported as confirmed or suspected cases of tuberculosis (TB). Overall, isolates of Mycobacterium tuberculosis of 361 patients $(90 \%$ of the bacteriologically confirmed cases) were available to study the frequency of transmission of the strains on a molecular level.

Restriction fragment length polymorphism (RFLP) analysis was performed by using IS6110 and the polymorphic GC-rich sequence (PGRS) as genetic markers.

Ninety nine isolates shared by 77 patients $(21.3 \%)$ were associated with 28 IS6110defined clusters. However, secondary typing of low copy number isolates decreased the number of clusters to 25 , encompassing 81 isolates from $63(17.5 \%)$ patients. By deoxyribonucleic acid (DNA) fingerprinting plus conventional contact tracing, definite transmission of TB was proven in only five patients $(1.4 \%)$ and assumed in 20 patients $(5.6 \%)$. In all other cluster-associated isolates, no epidemiological connections between the patients could be found using the clinical and sociodemographic data available.

The present study demonstrates that in the time period studied only minor transmission occurred.

Eur Respir J 1998; 11: 804-808.
*Swiss National Center for Mycobacteria, Dept of Medical Microbiology, University of Zurich, 8028 Zurich, Switzerland. +Zürcher Höhenklinik Wald, 8639 Faltigberg, Switzerland.

Correspondence: G.E. Pfyffer

Swiss National Center for Mycobacteria

Dept of Medical Microbiology

University of Zurich

Gloriastrasse 30

8028 Zurich

Switzerland

Fax: 4116344918

Keywords: Deoxyribonucleic acid fingerprinting, insertion sequence, molecular epidemiology, restriction fragment length polymorphism, transmission, tuberculosis

Received: October 271997

Accepted after revision January 201998

Supported by the Belport Foundation (Zurich), the Zurich Lung Association, and the Swiss Federal Office of Public Health.
Restriction fragment length polymorphism (RFLP) analysis of Mycobacterium tuberculosis based on the insertion sequence IS6110 has recently been standardized and become an important molecular tool in answering questions such as: 1) transmission of the disease in community $[1,2]$ and international settings [3] as well as via the nosocomial route $[4,5] ; 2)$ reactivation versus reinfection of tuberculosis (TB) [6]; and 3) cross contamination of cultures in the laboratory [7].

Large population-based studies undertaken in New York [8] and San Francisco [9] utilizing IS6110-based RFLP analyses have revealed clusters, which inferred that recen-tly transmitted TB encompassed $30-40 \%$ of TB cases. Even though these large urban areas have a high proportion of patients infected with human immunodeficiency virus (HIV), who are known to be more susceptible to in-fections and at greater risk of latent TB infection rapidly progressing to active disease, these figures greatly exceed general assumptions regarding transmission $(\sim 10 \%$; [10]). Both studies concentrating on these big cities are based on the assumptions that deoxyribonucleic acid (DNA) fingerprinting using the IS6110 sequence as a probe provides ac-curate typing of $M$. tuberculosis isolates and, furthermore, that the identification of cluster strains necessarily implies recent transmission.

Most recent concepts of molecular epidemiology utilizing additional genetic markers, such as the polymorphic

For editorial comments see page 795
GC-rich repetitive sequence (PGRS; $[11,12])$ and/or the 36 base pair (bp) direct repeat (DR; $[13,14]$ ) have, however, demonstrated that DNA fingerprinting with the IS6110 technique was not always sufficient, particularly, in those cases where isolates have fewer than six copies of IS6110 [15-17]. Use of supplementary fingerprinting techniques markedly decreased clustering and, thus, put the transmission links suggested by molecular biology more in line with epidemiological data.

In the present study RFLP patterns of $M$. tuberculosis strains isolated from TB patients of the metropolitan area of Zurich (population 1.2 million; area: $1,729 \mathrm{~km}^{2}$ ) over a period of 3 yrs were analysed to determine the frequency of recent transmission, which eventually reflects the effectiveness of current measures of TB control, i.e., on case finding and management, contact tracing and preventive therapy strategies.

\section{Methods}

\section{Patient population}

The study cohort included 444 patients of the Canton of Zurich, all being treated for TB between 1991 and 1993 (278 males, 166 females; 216 Swiss-born (48.6\%), 228 foreign-born $(51.4 \%)$ ). 


\section{Mycobacterial isolates}

Within the cohort of bacteriologically confirmed TB, isolates of M. tuberculosis were available from 361 (90.0\%) patients. The majority of these $(291(80.6 \%))$ had one isolate, $54(15.0 \%)$ had two isolates, $13(3.6 \%)$ had three isolates, one patient $(0.3 \%)$ had five isolates, and two patients $(0.6 \%)$ had eight isolates each. DNA fingerprinting was performed on all available isolates $(\mathrm{n}=461)$. Clinical specimens included sputa, bronchial aspirates or washings, blood, cerebrospinal fluids, pleural aspirates, gastric and pericardial fluids, urine, faeces and pus. Cultures on Löwenstein-Jensen slants (produced in the Department of Medical Microbiology), on Middlebrook 7H10/sel 7H11 agar (biplates; Becton Dickinson Microbiology Systems, Cockeysville, MD, USA), and in BACTEC 460 medium (12B and 13A; Becton Dickinson Diagnostic Instrument Systems, Sparks, MD, USA), as well as identification procedures, were performed as described earlier [18]. Isolates were kept at $-80^{\circ} \mathrm{C}$ and subcultured thereafter for DNA fingerprinting.

\section{RFLP analysis}

DNA fingerprinting based on IS6110 was performed according to the standardized protocol of VAN EMBDEN et al. [19] with modifications [15]. The genetic identity of $M$. tuberculosis strains which exhibited: 1) a low number of IS6110 copies; or 2) in rare instances, very minor differences in the position of one band were confirmed by using an additional probe: A $l u \mathrm{I}$ digested chromosomal DNA was hybridized with a PGRS (EcoRI-HindIII fragment isolated from plasmid pTBN12; [12]). The IS6110 and the PGRS probe had been labelled with the DIG High Prime Labelling Kit. Banding patterns were detected by the DIG Luminescent Detection Kit. Restriction enzymes, nylon membranes and labelling and detection kits were purchased from Boehringer (Mannheim, Germany).

\section{Comparison of RFLP patterns}

Using a HP ScanJet 4c (Hewlett Packard, Greeley, CO, USA; scanned at 190 dots per inch (dpi)) IS6110 patterns were analysed by GelCompar software (Windows 95, version 4.0; Applied Math, Kortrijk, Belgium). The mobility of the IS6110 fragments was compared to the molecular weight marker $\lambda$-DNA EcoRI/HindIII (Boehringer) which was used as an external size marker. In addition, the accuracy of the procedure was tested by comparing the IS6110 banding pattern of the TB strain Mt14323 (a gift from J.D.A. van Embden) which was present on each autoradiograph. Following the instructions of the Gelcompar manufacturer, patterns were compared by the unweighted pair group method with averages (UPGMA) clustering method using the Dice coefficient (1.2\%). Banding patterns obtained after hybridization with the PGRS probe were visually evaluated. To facilitate visual reading of PGRS fingerprints, samples were electrophoresed together on the same gel according to the results with IS6110. As suggested by Burman et al. [17] only fragments exceeding $1.6 \mathrm{~kb}$ were analysed.

\section{Definition of clustering}

A "cluster" of $M$. tuberculosis isolates was defined as two or more isolates which either: 1) exhibited the same number of copies (six or more) with identical molecular weights of the IS6110 fragments; or 2) revealed five or fewer IS6110 fragments of the same molecular weight and identical PGRS RFLP patterns.

\section{Epidemiological investigation}

All patients in the cohort with available isolates of $M$. tuberculosis $(\mathrm{n}=361)$ were evaluated. Clinical aspects (disease localization and type, date of TB diagnosis, HIV status), sociodemographic data (such as nationality, place of birth, home address at time TB was diagnosed, profession) and additional patients' aspects (e.g., homelessness, i.v. drug abuse (IVDA), alcohol abuse, psychiatric illness, etc.) which have been compiled previously [20] were reviewed to look for any apparent epidemiological links. Results of conventional epidemiological contact tracing were available for those patients centrally investigated by the Zurich Lung Association. Among the patients associated with an IS6110-based cluster we defined patients as having: 1) "definite" transmission patterns if strains were identical upon DNA fingerprinting and there were epidemiological links upon contact tracing; 2) "assumed" transmission patterns if DNA fingerprints were identical and possible contacts were very likely, though unnoticed, as based on the available sociodemographic data; and 3) "unidentifiable" transmission patterns if strains displayed identical RFLP patterns, but no clear links could be found in conventional contact tracing.

\section{Results}

\section{Study population and clinical isolates of the cohort}

During the 3 yr study period (1991-1993) 444 patients in the Zurich metropolitan area were reported to the local and federal health authorities as confirmed or suspected cases of active TB. Three hundred and ten patients $(69.8 \%)$ had pulmonary TB, $90(20.2 \%)$ extrapulmonary $\mathrm{TB}$, and $44(10 \%)$ had pulmonary as well as extrapulmonary disease. Of the 444 TB patients, 401 cases (90.3\%) were bacteriologically confirmed. Eventually, M. tuberculo-sis isolates were available for DNA fingerprinting from 361 patients (i.e., $90.0 \%$ of the culture-positive cases). Since some patients had multiple isolates (two to eight) there were a total of 461 isolates which eventually underwent DNA fingerprinting. All strains tested belonged to the $M$. tuberculosis complex (verified by gene probes) and were biochemically identified as $M$. tuberculosis by their negative catalase reaction and positive nitrate and niacin tests.

RFLP analysis of M. tuberculosis using IS6110 as a genetic marker

Hybridization of PvuII-digested chromosomal DNA with the 245 bp fragment of IS6110 resulted in highly variable RFLP patterns. The degree of relatedness among the isolates (i.e., the similarity of the DNA fingerprints) was 
assessed by a dendrogram generated by computer analysis (data not shown). A total of 318 different RFLP patterns was observed, whereby the number of IS6110 copies per isolate of M. tuberculosis ranged 1-22. Six or more copies were found in $343(93 \%)$ of the isolates. Among the 361 patients there were 70 individuals of whom multiple isolates (two to eight) had been available for typing. In most of these cases, the number of IS6110 copies and integration sites remained generally unchanged over the $3 \mathrm{yr}$ period, regardless of emerging drug resistance in some of the cases. There were six notable exceptions, where a loss or an increase in IS6110 copies was observed (one to two bands).

Isolates with matching IS6110 patterns were considered to comprise a cluster of epidemiologically associated strains. A total of 28 IS6110 banding patterns, each exhibiting a characteristic number of copies and well-defined fragment lengths, were shared by 77 patients with 99 isolates. There were two clusters harbouring six patients each, one cluster with five patients, three clusters with four patients, four clusters with 3 patients, and 18 clusters harbouring two patients each. An inverse correlation was found between the number of IS6110 copies per isolate and the likelihood of matching of fingerprint patterns: 12 (70.6\%) of 17 patients' strains displaying one to three IS6110 copies and $87(25.3 \%)$ of 344 patients' strains having more than six copies of IS6110 were clustered.

\section{Secondary typing}

Secondary typing of strains with a low number of IS6110 copies ( $ð 5$ bands) revealed that cluster 28 consisted of two instead of six patients (fig. 1) and cluster 27 of three instead of four patients. Clustered strains exhibiting more than five bands were also probed with the PGRS when very minor differences in the position of one single band occurred. This confirmed strain identity within cluster numbers 1,25 (fig. 2 ) and 26, but not within clusters 2 , 3 and 8. After eliminating isolates with identical IS6110 RFLP patterns, but unique PGRS fingerprints, the number of clusters decreased from 28 to 25 and the number of patients from 77 to 63 .

\section{Assessment of possible transmission patterns}

Clusters of low copy number strains (clusters 27 and 28 with three and one IS6110 copies, respectively) could only be partially verified by secondary typing. Cluster 27 finally consisted of three patients instead of four, all males of the same age group, with IVDA and HIV infection as the most predominant risk factors (isolates being resistant to isoniazid, ciprofloxacin, and ofloxacin). Cluster 28 (fig. 1) ended up consisting of only two patients, both originating from Sri Lanka, while the other four patients with identical IS6110 but distinct PGRS patterns were from Sri Lanka (one), India (two), and Vietnam (one). Identical patterns were obtained by secondary typing for some clusters with seven and eight bands, namely numbers 1,25 and 26 . In cluster 25 there was one patient with an IS6110 pattern that differed in one band from that of the other three patients. When typed with the PGRS, all four pati-ents' isolates showed the same banding patterns (fig. 2). In the three IS6110-defined clusters with divergence in sec-ondary typing (No.s 2, 3 and 8) no epidemiological links could be found. As regards the IS6110-defined clusters a)

$\begin{array}{lllllll}M & 1 & 2 & 3 & 4 & 5 & 6\end{array}$

b)

21.2

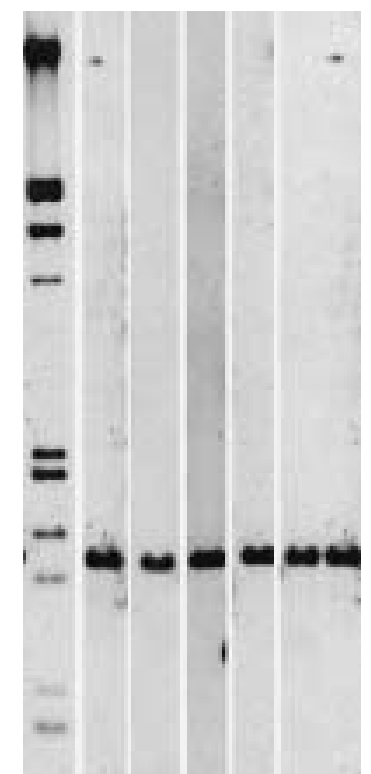

M 121230456

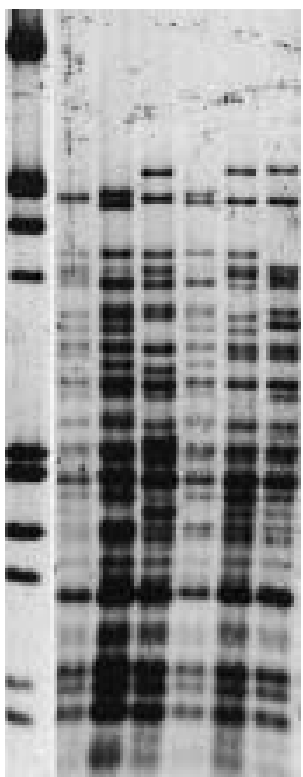

Fig. 1. - Genomic fingerprints of six Mycobacterium tuberculosis isolates (exhibiting one single IS6110 copy) of an assumed cluster (No. 28) of which only two strains eventually turned out to be cluster strains. a) lanes 1-6: PvuII-digested chromosomal deoxyribonucleic acid (DNA) probed with IS6110 (identical patterns in all lanes); b) AluIdigested chromosomal DNA probed with the polymorphic GC-rich sequence (PGRS) (lanes 2 and 4: identical patterns; lanes 1, 3, 5 and 6: unique patterns). M: molecular weight marker (EcoRI-HindIII-digested bacteriophage $\lambda$ DNA).

a)

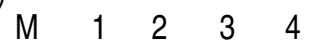

b)
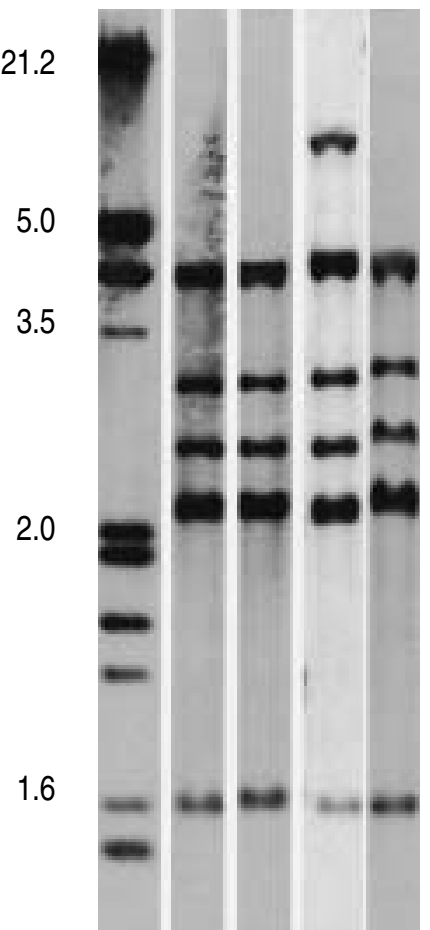

M
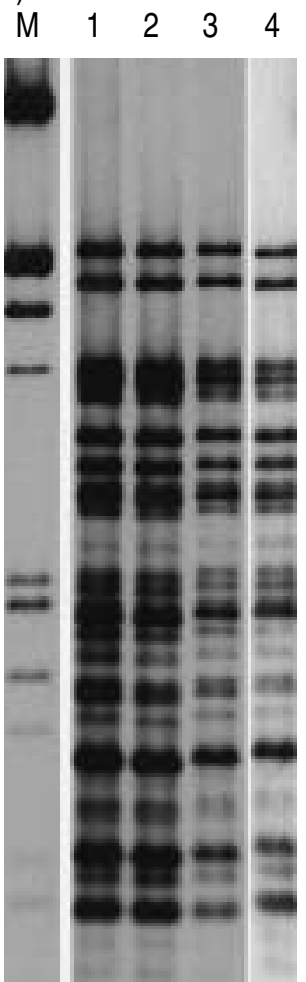

Fig. 2. - Genomic fingerprints of clonal Mycobacterium tuberculosis isolates (cluster no. 25). a) lanes 1-4: PvuII-digested chromosomal DNA probed with IS6110 (lanes 1, 2 and 4: identical patterns; lane 3: isolate with one additional band); b) lanes 1-4, AluI-digested chromosomal DNA probed with the PGRS (identical patterns in all lanes). M: molecular weight marker. 
with a high number of copies ( $>9$ ), clusters $6,11,15,16$ and 22 consisted predominantly of persons known as alcohol abusers, homeless and/or living in the same town, or having the same country of origin. In cluster 19 we have found two isolates which originated from two patients having, at first sight, no links. However, one of the patients was a laboratory technician who had acquired TB via needle puncture while handling the cultures of the other patient in the laboratory. No epidemiological links were identifiable for patients within clusters $4,7,9,10$, $14,17,18,20$ and 21.

Within the clusters found in this study there were eventually five patients (clusters 19 and 27) with definite and 20 patients (clusters $6,11,13,15,16,22,23$ and 28) with assumed transmission patterns. For the remaining 38 patients $(58.7 \%)$ associated in 15 clusters, transmission patterns were not identifiable. A comprehensive list of all clusters can be obtained from the corresponding author.

\section{Discussion}

In our cohort analysis over a 3 yr period (1991-1993) 461 M. tuberculosis isolates from 361 TB patients were characterized by RFLP. These patients lived in the metropolitan area of Zurich, which covers merely $4.2 \%$ of the country's area, but is home to $20 \%$ of the total population of Switzerland and accounts for $\sim 25 \%$ of all TB cases. Patients were managed by a primary care physician-based TB treatment programme. Sociodemographic and clinical data, including risk factors for TB, were compiled to assess the effectiveness of this kind of treatment programme [20]. Factors negatively associated with compliance to therapy were prevalent in $172(38.7 \%)$ patients and consisted of asylum seeking (and, thus, frequent change of address) in $10.1 \%$, alcoholism in $9.7 \%$, psychiatric ailments in $8.6 \%$, unemployment in $6.3 \%$, drug abuse in $5.4 \%$ and homelessness in $2.9 \%$. HIV testing was performed in 160 patients; 42 persons $(9.5 \%)$ reported their tests as positive.

Identical RFLP patterns were found in the majority of the strains of the present patients $(91.5 \%)$, from whom multiple isolates were available over a period of 1-3 yrs. The DNA fingerprint data generated by comparing the earlier isolates with the more recent ones displayed the known degree of stability of the IS6110 element over extended periods of time, regardless of emerging drug resistance $[15,21,22]$. In only six cases (all isolates having $>9$ bands) was a loss or an increase of one to two insertion copies observed

Strains of M. tuberculosis are considered to be related at the genetic level when isolates exhibit the same fragment length and the same number of copies of IS6110, but may also be related even if they differ in the position or presence of one or two IS6110 fragments making up the DNA fingerprint [22], as may occur due to transposition and recombination. Recent, large population-based studies have shown these assumptions, however, to be on weak grounds, particularly, when strains with five or fewer copies are fingerprinted [16, 17]. Our study largely confirms these recent findings that coincidental IS6110 matches are not uncommon. These findings suggest that, in many of the large population-based studies, the number of clusters and hence, degree of recent transmission, were overestimated.
On the basis of the IS6110 copy number and fragment length, 99 isolates from 77 patients were tentatively grouped into 28 clusters. After secondary typing 13 patients from three of the original 28 clusters were excluded, leaving 25 clusters comprising 63 patients $(68.2 \%$ Swiss-born, $27.0 \%$ foreign-born, $4.8 \%$ with unknown nationality).

In contrast to the study of BRADEN et al. [16] (where over $33 \%$ of the isolates were not available for typing) only $10 \%$ of the isolates of bacteriologically confirmed cases were missing in our study. Nonavailability of isolates and of clinical and sociodemographic data was due to the commonly encountered difficulties when performing retrospective studies: 1) some strains may fail to grow after having been kept in a culture collection; 2) tubercle bacilli, isolated and identified by other laboratories, were not always saved; 3 ) some patients could not be traced due to death or emigration; and 4) some clinicians and patients refused to be interviewed.

Even though a high proportion of patients' isolates and detailed clinical and sociodemographic data were available, conventional contact tracing fell far short of detecting many of the transmission links postulated by molecular biology. Only in two clusters was transmission among their members definite (clusters 19 and 27). In some others (clusters 6, 11, 15, 16 and 22) transmission was judged as very probably due to common risk factors for $\mathrm{TB}$, such as substance abuse (clusters being found in $48.8 \%$ of alcoholics and $20.8 \%$ of IVDA) or homelessness (clustering occurred in $61.5 \%$ ) (table 1). Therefore, our data parallel those of BRADEN et al. [16] and BuRMAn et al. [17] who have demonstrated in population-based studies in Colorado and Arkansas, respectively, that substance abuse and homelessness were among the most significant risk factors contributing to the transmission of TB. Infection with the same strain of $M$. tuberculosis as suggested by molecular biology in clusters $11,13,16$ and 27 was among persons all living in the same town (population $\breve{S} 10,000$ ) or village (population $<10,000$ ) and for patients in clusters 22 and 28 of the same country of origin. In a significant number of clusters $(58 \%$; mostly including people from totally different social groups and individuals living far apart from each other) we were unable to determine possible transmission links, probably because transmission occurred unnoticed.

Table 1. - Patient characteristics with certain and possible epidemiological links

\begin{tabular}{|c|c|}
\hline Characteristics & Value \\
\hline Average age yrs (range) & $48.8(10-88)$ \\
\hline Gender $\mathrm{M} / \mathrm{F}$ & $46 / 17$ \\
\hline \multicolumn{2}{|l|}{ Nationality $\mathrm{n}(\%)$} \\
\hline Swiss-born & $43 \quad(68.2)$ \\
\hline Foreign-born & $20 \quad(31.8) *$ \\
\hline \multicolumn{2}{|c|}{ Major risk factors for TB (sometimes multiple)+ } \\
\hline Homeless & $8 / 13$ \\
\hline Alcoholic & $21 / 43$ \\
\hline IVDA & $5 / 24$ \\
\hline HIV-seropositive & $8 / 42$ \\
\hline Asylum seeker & $2 / 45$ \\
\hline Patients with no known risk factors & 27 \\
\hline
\end{tabular}

*: four from Italy and former Yugoslavia, three from Turkey and unknown, two from Pakistan and Sri Lanka, one from South Korea and Estonia; +: values represent the number of subjects at risk/total population. M: male; F: female; TB: tuberculosis; IVDA: $i$.v. drug abuser; HIV: human immunodeficiency virus. 
DNA fingerprinting can be considered a powerful tool to monitor the performance of TB control. Overall, the number of patients associated with clusters in this study (17.5\%) was even lower than that reported from areas with similarly little ongoing transmission (e.g., 33\% [16]; 26.9\% [17]). The reasons for this are manifold: 1) compared with other metropolitan areas such as New York or San Francisco, Zurich has a lower TB incidence and a lower rate of HIV infection [20]; 2) the Canton of Zurich has a high density of physicians, in 1993 amounting to one physician per 527 inhabitants, a ratio that is below the national average (one per 604 inhabitants; [23]); 3) TB surveillance is organized very strictly, i.e., at the national level through the Swiss Federal Office of Public Health, at the cantonal level by the cantonal health authorities and at the regional level by the local chapter of the lung association; 4) reporting of all cases of active TB to the Swiss Federal Office of Public Health is mandatory, both by the physician involved in the diagnostic work-up as well as by the mycobacteriology laboratory; 5) mycobacteriological analyses are performed free of charge in the Canton of Zurich and susceptibility testing is routinely performed in all cases of active TB, as a long-standing practice; 6) TB treatment is covered by health insurance (mandatory for all residents) and management of TB patients is carried out through a decentralized, primary-care physician based system; and 7) conventional contact tracing and implementation of directly observed therapy are performed by the local lung association in collaboration with the primary-care physician. Although this primary-care physician based system has been shown to have certain weaknesses [20], low levels of recent transmission, as demonstrated in this study, may suggest the overall effectiveness of TB control in the Canton of Zurich.

Acknowledgments: The authors would like to thank A. von Graevenitz for helpful suggestions and for reading the manuscript. On the occasion of their Annual Meeting in 1997, the Swiss Society of Pneumology awarded the Crivelli Prize to the authors of this study.

\section{References}

1. Genewein A, Telenti A, Bernasconi C, et al. Molecular approach to identifying route of transmission of tuberculosis in the community. Lancet 1993; 342: 841-844.

2. Kline S, Hedemark L, Davies S. Outbreak of tuberculosis among regular patrons of a neighbourhood bar. $N$ Engl $J$ Med 1995; 333: 222-227.

3. Kiers A, Drost AP, von Soolingen D, Veen J. Use of DNA fingerprinting in international source case finding during a large outbreak of tuberculosis in the Netherlands. Int $J$ Tuberc Lung Dis 1997; 1: 239-245.

4. Jereb JA, Burwen DR, Dooley SW, et al. Nosocomial outbreak of tuberculosis in a renal transplant unit: application of a new technique for restriction fragment length polymorphism analysis of Mycobacterium tuberculosis isolates. J Infect Dis 1993; 168: 1219-1224.

5. Daley C, Small P, Schechter G, et al. An outbreak of tuberculosis with accelerated progression among persons infected with the human immunodeficiency virus: an analysis using restriction-fragment-length polymorphisms. $N$ Engl J Med 1992; 326: 231-235.

6. Van Soolingen D, Hermans PWM. Epidemiology of tuberculosis by DNA fingerprinting. Eur Respir J 1995; 8: Suppl. 20, 649s-656s.
7. Small PM, McClenny NB, Singh SP, Schoolnik GK, Tompkins LC, Mickelsen PA. Molecular strain typing of Mycobacterium tuberculosis to confirm cross-contamination in the mycobacteriology laboratory and modification procedures to minimize occurrence of false-positive cultures. J Clin Microbiol 1993; 31: 1677-1682.

8. Alland D, Kalkut E, Moss AR, et al. Transmission of tuberculosis in New York City. An analysis by DNA fingerprinting and conventional epidemiologic methods. $N$ Engl J Med 1994; 330: 1710-1716.

9. Small PM, Hopewell PC, Singh SP, et al. The epidemiology of tuberculosis in San Francisco. A population-based study using conventional and molecular methods. $N$ Engl J Med 1994; 330: 1703-1709.

10. Horvitz O, Edwards PO, Lowell AM. National tuberculosis control program in Denmark and the United States. Health Serv Rep 1973; 88: 493-498.

11. Poulet S, Cole ST. Characterization of the highly abundant polymorphic GC-rich-repetitive sequence (PGRS) present in Mycobacterium tuberculosis. Arch Microbiol 1995; 163: 87-95.

12. Ross BC, Raios K, Jackson KM, Dwyer B. Molecular cloning of a highly repeated DNA element from Mycobacterium tuberculosis and its use as an epidemiological tool. J Clin Microbiol 1992; 30: 942-946.

13. Hermans PWM, van Soolingen D, Bik EM, de Haas PEW, Dale JW, van Embden JDA. Insertion element IS987 from Mycobacterium bovis BCG is located in a hot-spot integration region for insertion elements in Mycobacterium tuberculosis complex strains. Infect Immun 1991; 59: 2695-2705.

14. Poulet S, Cole ST. Repeated DNA sequences in mycobacteria. Arch Microbiol 1995; 163: 79-86.

15. Strässle A, Putnik J, Weber R, Fehr-Merhof A, Wüst J, Pfyffer GE. Molecular epidemiology of Mycobacterium tuberculosis strains isolated from patients in a human immunodeficiency virus cohort in Switzerland. J Clin Microbiol 1997; 35: 374-378.

16. Braden CR, Templeton GL, Cave MD, et al. Interpretation of restriction-fragment-length polymorphism analysis of Mycobacterium tuberculosis isolates from a state with a large rural population. J Infect Dis 1997; 175: 1446-1452.

17. Burman WJ, Reves RR, Hawkes AP, et al. DNA fingerprinting with two probes decreases clustering of $\mathrm{Myco}$ bacterium tuberculosis. Am J Respir Crit Care Med 1997; 155: 1140-1146.

18. Pfyffer GE, Kissling P, Wirth R, Weber R. Direct detection of Mycobacterium tuberculosis complex in respiratory specimens by a target-amplified test system. J Clin Microbiol 1994; 32: 918-923.

19. Van Embden JDA, Cave MD, Crawford JT, et al. Strain identification of Mycobacterium tuberculosis by DNA fingerprinting: recommendations for standardized methodology. J Clin Microbiol 1993; 31: 406-409.

20. Rose N, Shang H, Pfyffer GE, Brändli O. Tuberkulosetherapie im Kanton Zürich 1991-1993: Was sind die Ursachen für Rezidive und Therapieversager? Schweiz. Med Wochenschr 1996; 126: 2059-2067.

21. Van Soolingen D, Hermans PWM, de Haas PEW, Soll DR, van Embden JDA. Occurrence and stability of insertion sequences in Mycobacterium tuberculosis complex strains: evaluation of an insertion sequence-dependent DNA polymorphism as a tool in the epidemiology of tuberculosis. J Clin Microbiol 1991; 29: 2578-2586.

22. Cave MD, Eisenach KD, Tempelton G, et al. Stability of the fingerprint pattern produced with IS6110 in strains of M. tuberculosis. J Clin Microbiol 1994; 32: 262-266.

23. Jau J. Mitgliederstatistik 1993 der Verbindung der Schweizer Ärzte. Schweiz Ärztezeitung 1994; 75: 756-773. 